\title{
Healthy and Pathological Cerebellar Spiking Neural Networks in Vestibulo-Ocular Reflex
}

\author{
Alberto Antonietti, Claudia Casellato, Alice Geminiani, Egidio D’Angelo, Alessandra Pedrocchi
}

\begin{abstract}
Since the Marr-Albus model, computational neuroscientists have been developing a variety of models of the cerebellum, with different approaches and features. In this work, we developed and tested realistic artificial Spiking Neural Networks inspired to this brain region. We tested in computational simulations of the Vestibulo-Ocular Reflex protocol three different models: a network equipped with a single plasticity site, at the cortical level; a network equipped with a distributed plasticity, at both cortical and nuclear levels; a network with a pathological plasticity mechanism at the cortical level. We analyzed the learning performance of the three different models, highlighting the behavioral differences among them. We proved that the model with a distributed plasticity produces a faster and more accurate cerebellar response, especially during a second session of acquisition, compared with the single plasticity model. Furthermore, the pathological model shows an impaired learning capability in Vestibulo-Ocular Reflex acquisition, as found in neurophysiological studies. The effect of the different plasticity conditions, which change fast and slow dynamics, memory consolidation and, in general, learning capabilities of the cerebellar network, explains differences in the behavioral outcome.
\end{abstract}

\section{INTRODUCTION}

In computational neuroscience, the cerebellum is one of the central nervous system structures that has been studied in more details, since it has a clear and organized anatomical structure and for its crucial role in motor learning. The cerebellum is capable of expressing learning in both temporal association of discrete behavioral responses and in generation of voluntary and reflex movements [1]-[3]. In motor adaptation, it is supposed to work as a predictive controller and its learning capabilities are ascribed to longterm synaptic modifications: Long-Term Depression (LTD) and Long-Term Potentiation (LTP) [4]. These synaptic changes involve different synaptic connections, at various levels of the cerebellar circuit. In particular, LTP and LTD at Parallel Fibers/Purkinje Cells (PF-PC) are the first mechanisms driving the output modulation: thanks to the Climbing Fibers (CFs) teaching signal, PF-PC synapses

* This work was supported by grants of European Union: CEREBNET FP7-ITN238686, REALNET FP7-ICT270434, Human Brain Project HBP604102

A. Antonietti, C. Casellato, A. Geminiani and A. Pedrocchi are with the Neuroengineering and Medical Robotics Laboratory, Department of Electronics, Information and Bioengineering, Politecnico di Milano, P.zza L. Da Vinci 32, 20133, Milano, Italy (telephone: +39-02-2399-9509 e-mail: claudia.casellato@polimi.it).

E. D'Angelo is with the Brain Connectivity Center, IRCCS Istituto Neurologico Nazionale C. Mondino and the Department of Brain and Behavioral Sciences, University of Pavia, Via Forlanini 6, 27100, Pavia, Italy undergo the proper modifications in order to increase or decrease inhibition on Deep Cerebellar Nuclei (DCNs) having them to fire in a specific time window. However, multiple other plasticity sites coexist in the cerebellum. In particular, plasticities at nuclear sites, like Mossy FibersDeep Cerebellar Nuclei (MF-DCN) or Purkinje Cells-Deep Cerebellar Nuclei (PC-DCN) synapses, are responsible for a slow consolidation and refinement process, occurring through interaction and memory transfer from cerebellar cortex to the nuclei [5], [6].

One of the typical protocols involving the learning capability of the cerebellum is the Vestibulo-Ocular Reflex (VOR). During the VOR, the subject, animal or human, learns to move his eyes in order to compensate head rotations and to keep the image of target fixed on the retina. The cerebellum, in particular the flocculus, is supposed to tune the VOR, as emerged by lesion, pharmacological inactivation and genetic disruption studies [7].

Since the first version of the Marr-Albus Adaptive Filter Model [2], [3], computational neuroscientists have been developing a large variety of cerebellar models with an increasing number of realistic features. Spiking Neural Network (SNN) models represent a well-tested way to reproduce the cerebellar learning behavior. Very recently, Casellato et al. developed and tested a SNN-based cerebellar model in different tasks, including the VOR, both in simulations and embedded into a robotic controller [8]. This model has shown its effectiveness exploiting only the PF-PC plasticity. The aim of this work is to improve this model with the introduction of two plasticity sites at the nuclear level, in particular LTP and LTD at MF-DCN and PC-DCN connections. We tested the models in computational simulations reproducing the VOR protocol with two consecutive sessions, including acquisition and extinction phases. We expect to highlight the behavioral differences between the SNN model with one plasticity and the model with three plasticities. The introduction of nuclear plasticity sites, which have a slow timescale, should model consolidation mechanisms, detectable mainly in the reacquisition phase [9].

Finally, we manipulated the three-plasticity model to reproduce a pathological condition. Studies of VOR adaptation in mice, reporting damaged LTD at PF-PC synapses [10], showed that, if LTD at this plasticity site is blocked, the result is a severe damage to VOR adaptation, which can be ascribed to this alteration of low-level neural mechanism. We translated this impairment modifying the correspondent parameter in our SNN, expecting to reproduce impaired behavior in the VOR task execution. 


\section{MATERIALS AND METHODS}

\section{A. Cerebellar Model and Learning Rules}

The SNN used in computational simulations was composed of 2160 Leaky Integrate\&Fire neurons (Fig. 1).

- 100 Mossy Fibers (MFs), which encoded the vestibular information. Half of the MFs encoded the head angle and the other half encoded the head angular velocity.

- 2000 Granular Cells (GRs), which generated a sparsecoding representation of the input.

- 24 Inferior Olive cells (IOs), which encoded the gaze error (i.e. the difference between the desired and the actual direction of the eyes). Each IO is connected with one $\mathrm{PC}$ through one $\mathrm{CF}$.

- 24 Purkinje Cells (PCs), which had inhibitory connections to the DCNs. Each PC was randomly connected with the $80 \%$ of the GRs through PFs.

- 12 DCNs, which were the output of the cerebellar circuit, generating the motor command responsible for the eye rotation. Each DCN receives excitatory connections from all $100 \mathrm{MFs}$ and 2 inhibitory connections from 2 PCs.

Here the learning rules are described:

$\mathbf{1}^{\text {st }}$ learning rule: $\mathrm{PF}-\mathrm{PC}$

The first learning rule involved PF-PC connections and generated heterosynaptic LTP and LTD. Depending on the triggering signal from IOs (carried by CFs), LTD occurred at PF-PC connections corresponding to PFs that were active before the IO spike arrival. The maximum amount of LTD $(=-0.5878)$ was applied to the PFs which were active $100 \mathrm{~ms}$ before the IO spike. This time interval was in according to physiological reference values, in order to have the effect of plasticity and consequently the motor command with the proper anticipated timing, considering both the delays in neural and motor circuits. Otherwise, if a PF fired without the simultaneous firing of an IO, the corresponding synapse underwent a constant LTP $(=0.0073)$. Details can be found in [11].

$2^{\text {nd }}$ learning rule: MF-DCN

This plasticity rule strengthened and weakened MF-DCN synapses, by heterosynaptic LTP and LTD, triggered by PCs spikes. Specifically, if a MF and a PC fired within the same narrow time window, LTD was induced to the corresponding MF-DCN connections; otherwise, if a MF fired in absence of PCs activity, LTP occurred. The amount of LTD depended on the time interval between the MF and PC spikes, with a maximum $\left(=-6.1491 \cdot 10^{-8}\right)$ when the activity of MFs and PCs was synchronous, otherwise the amount of LTP was constant $\left(=1.4763 \cdot 10^{-5}\right)$. Details can be found in [12].

\section{$3^{\text {rd }}$ learning rule: $P C-D C N$}

This learning rule was a standard Spike-Timing Dependent Plasticity (STDP) [13]: when one of the two PCs (presynaptic) fired and soon after the corresponding DCN fired (within a LTP-time window $=20 \mathrm{~ms}$ ), the two inhibitory synapses from PCs to that DCN were increased (maximum LTP change: $\alpha_{3}=3.3874 \cdot 10^{-5}$ ). Otherwise, if the opposite chronological order occurred (within a pre-defined LTDtime window $=60 \mathrm{~ms}$ ), the synapses underwent LTD (maximum LTD change $\beta_{3}=1.8931 \cdot 10^{-7}$ ).

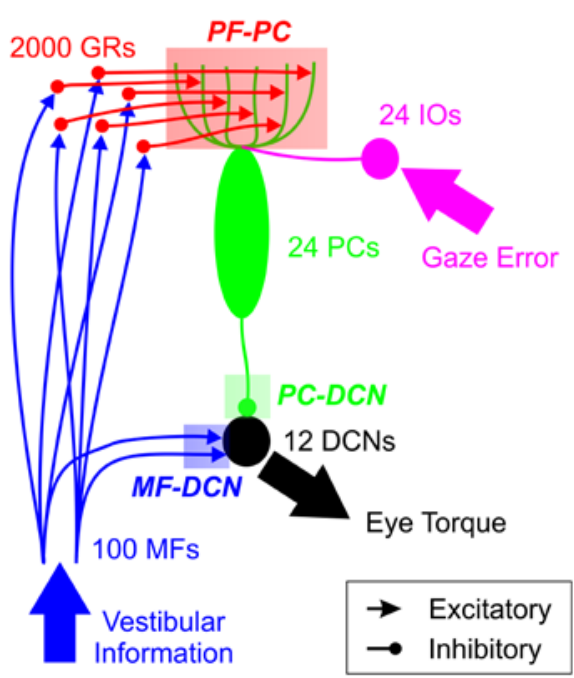

Figure 1. Cerebellar Model and Plasticity Sites

Topology of the SNN models used, with different groups of cells and the correspondence input/output variables. Transparent areas indicate the three plasticity sites: in red the PF-PC plasticity, in blue the MF-DCN plasticity and in green the PC-DCN plasticity.

The used LTP and LTD constants for the different learning rules come from a parameter optimization made by a Genetic Algorithm [14]. The one-plasticity model embedded only the first learning rule, the three-plasticity model embedded all the three learning rules, whereas the pathological model embedded three learning rules, with an impaired LTD at cortical level, modeled as a decrease of LTD $_{1}$ constant, set to the $30 \%$ of the optimal value.

To test the cerebellar model, we exploited the EventDriven simulator based on Look-Up-Tables [15], an opensource neural simulator which reduces the computational load using look-up tables. The simulations were run on a Dell Precision T3610, equipped with an Intel ${ }^{\circledR}$ Xeon ${ }^{\circledR}$ Processor E5-1620 v2 at $3.70 \mathrm{GHz}$ and $32 \mathrm{~GB}$ of RAM.

\section{B. Protocol}

The VOR protocol consisted of two sessions of 80 trials of acquisition and 20 trials of extinction. During the acquisition trials, the head performed a 2 seconds semi-sinusoidal rotation with an amplitude of $26^{\circ}$ at a frequency of $0.25 \mathrm{~Hz}$ and the eyes aimed at fixing a desired target. In order to maintain the gaze direction focused on the target, the cerebellum had to generate an eye counter-rotation with the same shape of the head but with the opposite sign. During the extinction trials, the head performed the same rotation, but the target was moving in the same direction of the head rotation. The cerebellum had to cancel the previously acquired behavior, since the eye counter-rotation was no more necessary to maintain the gaze on the target.

\section{Encoding and Decoding Strategies}

To communicate with the SNN, it was mandatory to define the encoding strategies, to transform input analog signals into cellular action potentials. The vestibular information (i.e. head position and velocity) was encoded using Radial 
Basis Functions to generate the MFs activity patterns, while the gaze error was encoded using a Poisson process that regulated the IOs activity [11]. The higher was the error, the higher was the IOs frequency, up to a maximum firing rate of $10 \mathrm{~Hz}$ [16]. We decoded the spiking activity of DCNs as an analog variable by a rate-based approach. We filtered the output variable with a 100 samples moving average filter, which simulated delay and smoothing in the motor peripheral circuit. The eye position was proportional to the cerebellar output torque $(1 \% \mathrm{mNm})$.

\section{Data Analysis}

For each of the three models, we computed: the Root Mean Square (RMS) gaze error for each trial, with the same sign of the median error within the trial; the VOR gain during the two acquisition phases, defined as the ratio between the amplitude of head and eye angular velocities; the number of spikes of PCs and DCNs within time-bins of $10 \mathrm{~ms}$ along the trial duration and along the different trials. We applied non parametric Kruskall-Wallis tests and post-hoc multiple comparison with Bonferroni correction to analyze the VOR gain in the two acquisition phases and in the three conditions; we set the significance level $p$ to 0.01 .

\section{RESULTS}

As a first result, the three different cerebellar models were able to learn to compensate the head rotation with a counterrotation of the eyes during the acquisition phases and to cancel the previously acquired behavior during the extinction phases. However, the capability of the model to express learning depended on its learning rules parameters. In fact the RMS gaze error with sign (Fig. 2) started from the same value $\left(-18.38^{\circ}\right)$ for all the three models, at the beginning of acquisition, but it evolved with different trends. The two healthy models were able, after a dozen of trials, to generate a cerebellar output that led to a fast decrease of the magnitude of the gaze error, towards lower values. The pathological model showed a much slower trend in error compensation, since it had a significant impairment of the LTD constant of the PF-PC plasticity, which is supposed to drive a fast tuning of the VOR. During the extinction phase, the error changed its sign, due to the so-called after-effect, but all the models were able to rapidly extinguish the

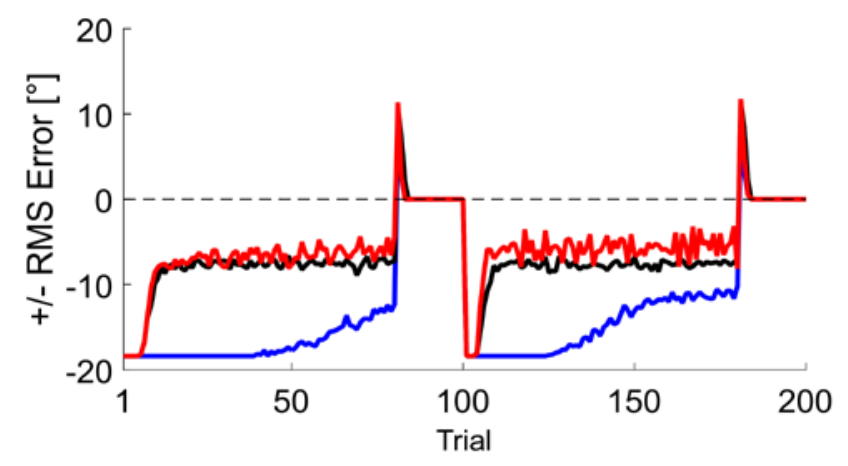

Figure 2. RMS Error comparison

RMS Gaze error (with sign) along trials in simulations with the oneplasticity model (in black), the three-plasticity model (in red) and the pathological model (in blue). unnecessary eyes rotation. During the second acquisition phase, it can be noticed that the three-plasticity model showed a faster and more effective response with respect to the one-plasticity model, which behaved as in the first acquisition phase. Indeed, the magnitude of the gaze error decreased to lower values with the distributed plasticity than with the cortical plasticity only. In addition, the pathological model, even if its error magnitude remained higher than the healthy models, showed a faster and stronger response in

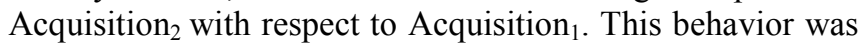
reflected by the velocity gain during the two acquisition phases (Fig. 3). The ideal gain is equal to one, meaning that the eye velocities have the same amplitude of the head velocity. The healthy models showed high gain values, in both sessions, whereas the pathological model was not able to generate a sufficient output, although improving during the second acquisition. The effect of multiple plasticity sites is clear from the analysis of neural activity (Fig. 4). In particular, in the one-plasticity model, there was no

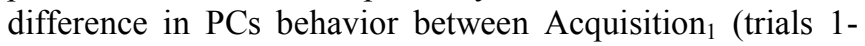
80) and Acquisition $_{2}$ (trials 101-180): learning was driven by PF-PC plasticity, which caused the PCs activity decrease in response to gaze error signal, thus allowing the corresponding DCNs to fire. The three-plasticity model acted in a different way thanks to the balance among multiple plasticity mechanisms: during Acquisition $_{1}$ both PCs and DCNs activity was similar to the one-plasticity behavior, but during Acquisition 2 the role of nuclear plasticity became evident. Learning was driven not only by PF-PC, but also by MF-DCN and PC-DCN plasticities. PCs activity was higher during Acquisition 2 with respect to Acquisition $_{1}$, since the output modulation was also the result of changes in the other nuclear synapses. The poor performance of the pathological model in Acquisition A was $_{1}$ due to a slower weakening of PCs, which continued to strongly inhibit DCNs. The behavioral improvement in Acquisition $_{2}$ was clearly due to the nuclear plasticity mechanisms that strengthened the excitation from MFs to DCNs and decreased the inhibitory influx of PCs to DCNs,

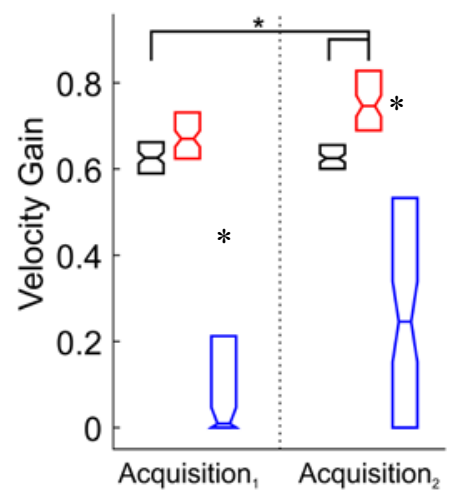

Figure 3. Velocity gain

Box plot of distribution of Velocity gain in the 80 trials of Acquisition and Acquisition ${ }_{2}$ in the three conditions: one-plasticity model (in black), the three-plasticity model (in red) and the pathological model (in blue). Asterisks indicate significant differences between groups $(p<0.01)$. Blue boxes are significantly different from all the other healthy boxes, but no significant difference come out between pathological gain in Acquisition $_{1}$ and Acquisition 2 . 

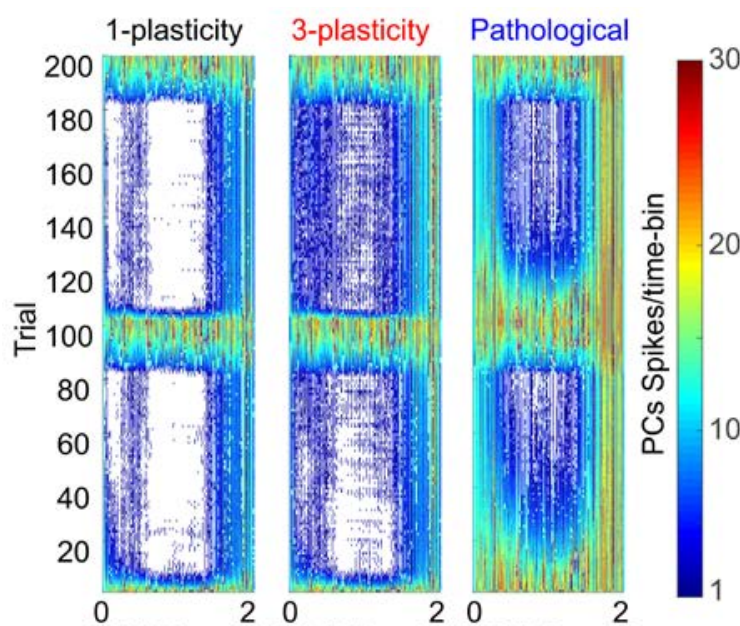

Trial Time [s] Trial Time [s] Trial Time [s]

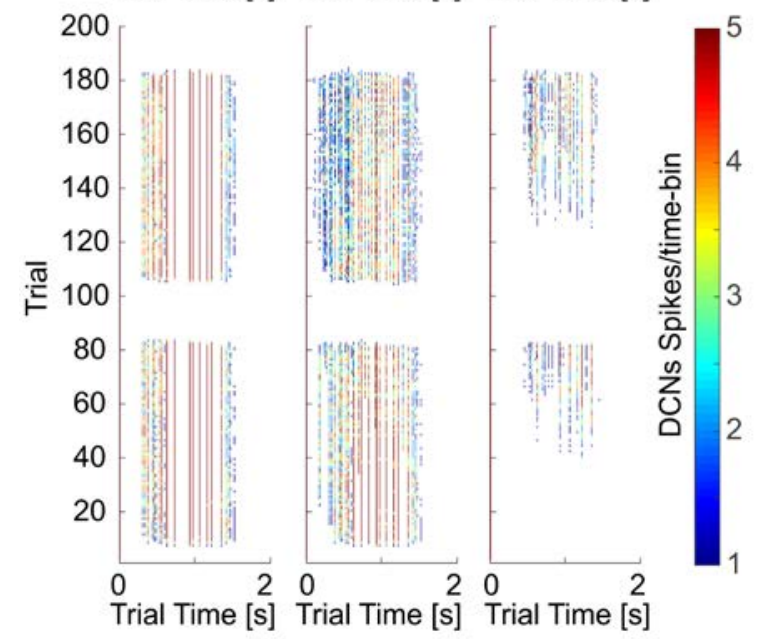

Figure 4. Spiking Activity of PCs and DCNs in single plasticity, distributed plasticity and pathological models

$P C s$ and DCNs spike distribution along trial time and along different trials. Each pixel represents one time-bin of $10 \mathrm{~ms}$, within it is computed the number of spikes of the correspondent group (first row PCs, second row DCNs). Each column represents a different model.

leading to an increased activity of DCNs, even if still compromised.

\section{CONCLUSION}

The obtained results demonstrated that the three different models showed learning capabilities to generate and extinguish the VOR. The differences in the output behavior were due to the different model parameters and reflected neurophysiological properties of cerebellar learning. The cortical plasticity was responsible for a fast acquisition, impaired in the pathological model, whereas the nuclear plasticities were responsible for slow adaptation and they modulated the DCNs activity. Nuclear plasticities increased the efficacy of cerebellar correction, allowing a memory transfer from the cortical to the nuclear layer. In conclusion, the three-plasticity model turned out to be the most effective and realistic and the pathological model demonstrated the same misbehavior of impaired cerebellum found in neurophysiological studies.

List of abbreviations

\begin{tabular}{ll}
\hline CFs & Climbing Fibers \\
DCNs & Deep Cerebellar Nuclei \\
IOs & Inferior Olive cells \\
LTD & Long-Term Depression \\
LTP & Long-Term Potentiation \\
MFs & Mossy Fibers \\
PCs & Purkinje Cells \\
PFs & Parallel Fibers \\
RMS & Root Mean Square \\
VOR & Vestibulo-Ocular Reflex \\
\hline
\end{tabular}

\section{REFERENCES}

J. C. Eccles, M. Ito, and J. Szentagothai, "The cerebellum as a neuronal machine," vol. 272, 1967.

[2] J. S. Albus, "A theory of cerebellar function," Math. Biosci., vol. 10, no. 1-2, pp. 25-61, Feb. 1971.

[3] D. Marr, "A theory of cerebellar cortex," J. Physiol., vol. 202, no. 2, pp. 437-70, Jun. 1969.

[4] E. D'Angelo, P. Mazzarello, F. Prestori, J. Mapelli, S. Solinas, P. Lombardo, E. Cesana, D. Gandolfi, and L. Congi, "The cerebellar network: from structure to function and dynamics.," Brain Res. Rev., vol. 66, no. 1-2, pp. 5-15, Jan. 2011.

J. A. Garrido, N. R. Luque, E. D’Angelo, and E. Ros, "Distributed cerebellar plasticity implements adaptable gain control in a manipulation task: a closed-loop robotic simulation," Front. Neural Circuits, vol. 7, no. October, p. 159, Jan. 2013.

[6] Y. Yang and S. G. Lisberger, "Role of plasticity at different sites across the time course of cerebellar motor learning," J. Neurosci., vol. 34 , no. 21 , pp. 7077-90, 2014.

[7] C. Burdess, "The vestibulo-ocular reflex: computation in the cerebellar flocculus," University of Edinburgh, 1996.

C. Casellato, A. Antonietti, J. A. Garrido, R. R. Carrillo, N. R. Luque, E. Ros, A. Pedrocchi, and E. D'Angelo, "Adaptive Robotic Control Driven by a Versatile Spiking Cerebellar Network," PLoS One, vol. 9, no. 11, p. e112265, Nov. 2014.

C. Casellato, A. Antonietti, J. A. Garrido, G. Ferrigno, E. D. Angelo, and A. Pedrocchi, "Distributed cerebellar plasticity implements generalized multiple - scale memory components in real - robot sensorimotor tasks," Front. Comput. Neurosci., vol. 9, Feb. 2015.

[10] C. I. De Zeeuw, C. Hansel, F. Bian, S. K. Koekkoek, A. M. van Alphen, D. J. Linden, and J. Oberdick, "Expression of a Protein Kinase C Inhibitor in Purkinje Cells Blocks Cerebellar LTD and Adaptation of the Vestibulo-Ocular Reflex," Neuron, vol. 20, no. 3, pp. 495-508, 1998.

[11] N. R. Luque, J. A. Garrido, R. R. Carrillo, S. Tolu, and E. Ros, "Adaptive cerebellar spiking model embedded in the control loop: context switching and robustness against noise," Int. J. Neural Syst., vol. 21, no. 5, pp. 385-401, Oct. 2011.

[12] A. Antonietti, C. Casellato, J. A. Garrido, E. D'Angelo, and A. Pedrocchi, "Spiking Cerebellar Model with Multiple Plasticity Sites Reproduces Eye Blinking Classical Conditioning," in Neural Engineering (NER), 2015 7th International IEEE/EMBS Conference on, 2015, pp. 296-299.

[13] N. Caporale and Y. Dan, "Spike timing-dependent plasticity: a Hebbian learning rule.," Аnnu. Rev. Neurosci., vol. 31, pp. 25-46, Jan. 2008.

[14] K. D. Carlson, J. M. Nageswaran, N. Dutt, and J. L. Krichmar, "An efficient automated parameter tuning framework for spiking neural networks," Front. Neurosci., vol. 8, no. February, p. 10, Jan. 2014.

[15] E. Ros, R. R. Carrillo, E. M. Ortigosa, B. Barbour, and R. Agís, "Event-driven simulation scheme for spiking neural networks using lookup tables to characterize neuronal dynamics," Neural Comput., vol. 18, no. 12, pp. 2959-93, Dec. 2006.

[16] E. D'Angelo, S. K. E. Koekkoek, P. Lombardo, S. Solinas, E. Ros, J. A. Garrido, M. Schonewille, and C. I. De Zeeuw, "Timing in the cerebellum: oscillations and resonance in the granular layer," Neuroscience, vol. 162, no. 3, pp. 805-15, Sep. 2009. 